\title{
I ncidental finding of a persistent sciatic artery aneurysm
}

\author{
Craig Nightingale ${ }^{1}$, Kapil Sahnan $^{1}$, Nadeem $_{\text {Qazi }}{ }^{2}$, Kaji Sritharan $^{1}$ \\ 1. Department of Vascular Surgery, St Mary's Hospital, London, United Kingdom. 2. Department of Interventional \\ Radiology, Charing Cross Hospital, London, United Kingdom.
}

Correspondence: Kapil Sahnan. Address: Department of Vascular Surgery, St Mary's Hospital, London, United Kingdom. Email: kapil.sahnan@nhs.net

Received: October 30, 2014

DOI : $10.5430 /$ crim.v2n1p92

Accepted: November 30, 2014 Online Published: December 15, 2014

URL: http://dx.doi.org/10.5430/crim.v2n1p92

\section{Abstract}

A 78 year old Afro Caribbean male, presented via accident \& emergency following a mechanical fall. Work-up investigations revealed an incidental $6.2 \mathrm{~cm}$ persistent sciatic artery aneurysm.

Following discussion with the vascular surgeons, lower limb angiography and a lower limb arterial duplex were performed. This demonstrated the true morphology of the persistent sciatic artery where it was shown to be the dominant blood supply to the leg; the superficial femoral artery in comparison was small and petered distally.

Treatment options were considered including embolization of the aneurysm and endovascular repair with a covered stent. Both options were considered too high risk for compromising the blood supply to the entire leg and the patient was managed conservatively.

A persistent sciatic artery (PSA) is a congenital anomaly, with an incidence estimated to be in the region of $0.03 \%-0.06 \%$. 5 Persistence of the embryological sciatic artery is rare and any patient with a PSA must be managed uniquely and in a considered manner. This case demonstrates the clinical significance of this anatomical variation and the potential pitfalls of treatment without adequate imaging of the lower limb. Patients with a patent sciatic artery are more prone to atherosclerotic degeneration and aneurysm formation, and this occurs in up to $44 \%^{[1]}$. Lack of recognition that the main blood supply to the lower leg is from a PSA may lead to inappropriate bypass grafting for what appears otherwise to be an occluded superficial femoral artery.

\section{Keywords}

Persistent sciatic system, Sciatic artery pseudoaneurysm

\section{Introduction}

Persistence of the embryonic sciatic artery is rare and any patient with a persistent sciatic artery (PSA) must be managed uniquely and in a considered manner. This case demonstrates the clinical significance of this anatomical variation and the potential pitfalls of treatment without adequate imaging of the lower limb. Patients with a patent sciatic artery are more prone to atherosclerotic degeneration and aneurysm formation, and this occurs in up to $44 \%^{[1]}$. Moreover, patients with a PSA are more likely to present with vascular symptoms than the general population and it is therefore worthwhile considering in all patients with peripheral vascular disease. Lack of recognition that the main blood supply to the lower leg 
is from a PSA may lead to inappropriate bypass grafting for what appears otherwise to be an occluded superficial femoral artery.

\section{Case presentation}

A 78 year old Afro Caribbean gentleman was bought into hospital by his wife after a mechanical fall onto his side. He did not sustain any injuries and had a history of mechanical falls due to poor mobility. He had a past medical history of recurrent falls, advanced vascular dementia, hypertension, left leg venous ulcers, deep vein thrombosis (DVT) 3 years ago, peptic ulcer disease and cataracts. His medication included anti-hypertensives, paracetamol and a proton pump inhibitor.

He lived at home with his wife who was his main carer and he required significant help with his activities of daily living. He was an ex-smoker and did not drink alcohol. There was no significant family history. Routine tests demonstrated a microcytic anaemia, with a haemoglobin of 51g/dl which likely contributed to his fall. A compturisted tomography (CT) scan of the brain excluded a stroke and oesophago-gastroduodenoscopy to look for a gastrointestinal source of bleeding demonstrated a gastric polyp only; this was excised. CT abdomen/pelvis showed diverticular disease and demonstrated a right PSA with a $6.2 \mathrm{~cm}$ pseudoaneurysm. This was confirmed on CT angiography of the abdomen and lower limb (see Figure 1, 2 and 3). The PSA was continuous with the popliteal artery in the leg, and the superficial femoral artery was of small calibre and did not extend past the mid-thigh. A diverticular bleed was deemed to be the cause of his anaemia and the finding of the $6.2 \mathrm{~cm}$ sciatic artery aneurysm was considered to be incidental. He was transfused with 4 units of blood and his haemoglobin remained stable during his inpatient stay.

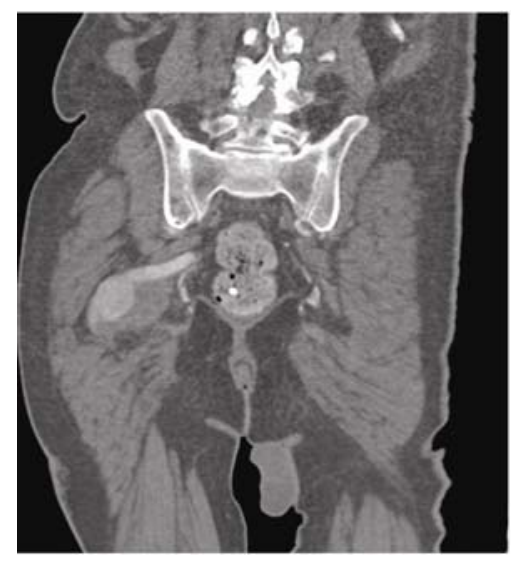

Figure 1. CT of PSA demonstrating thrombosed sac with pseudoaneurysm

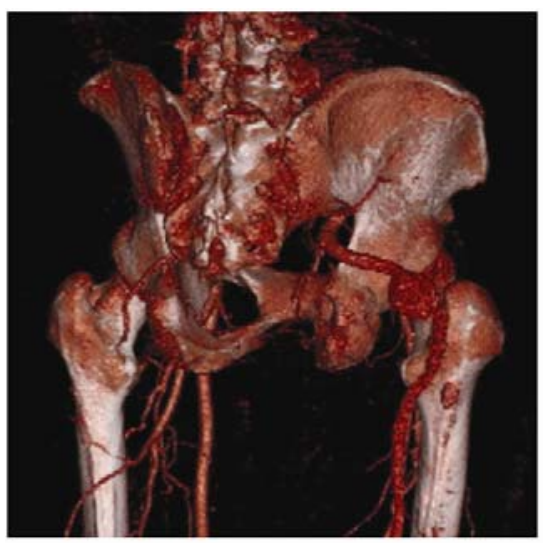

Figure 2. CT Reconstruction of PSA (posterior view)

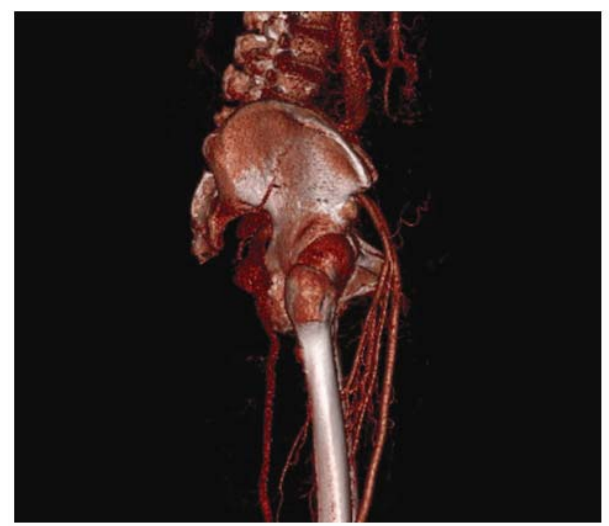

Figure 3. CT Reconstruction of PSA (lateral view) 
PSA aneurysms can cause acute arterial insufficiency due to thrombosis of the aneurysm or embolisation from a mural thrombus. Differential diagnosis includes an aneurysm of the gluteal artery, these are rare, and often occur as a result of pelvic trauma. Treatment options include embolization of the aneurysm, endovascular stenting or bypass grafting. Given that the PSA provided the main blood supply to the leg, embolization of the aneurysm and artery would render the leg ischaemic. Endovascular stenting of the aneurysm although feasible was associated with a risk of stent kinking which would also jeopardize the blood supply to the leg. The patient was high risk for a bypass operation under a general anaesthetic.

He was assessed by the geriatricians, physiotherapists and occupational therapists and the findings were discussed with the family. Given the patients overall condition and the potential risks with any endovascular option, and his unsuitability for general anaesthesia and a bypass procedure, the patient was managed conservatively. The joint vascular and interventional radiology MDT agreed with this assessment.

\section{Discussion}

Persistent sciatic arteries (PSA) are a very rare congenital abnormality, with an incidence estimated to be in the region of $0.03 \%-0.06 \%{ }^{[2]}$. It occurs due to failure of involution of the sciatic artery, and failure of the superficial femoral artery to develop. The PSA normally involutes during embryological development, as the superficial femoral artery establishes continuity with the PSA remnants - the popliteal and peroneal arteries. PSA has been reported in ages ranging from 6 months to 85 years (mean age 51 years) and has a slight male preponderance ${ }^{[3]}$. In a 1/3 of cases, its location is bilateral ${ }^{[4]}$.

These arteries may be termed "complete" when it forms the main blood supply to the lower leg (as in the majority), or incomplete when the superficial femoral artery is the main provider of blood to the popliteal artery ${ }^{[2]}$. Four different types of PSA have been described. In type 1, there is a complete PSA with a normal femoral artery; type 2 represents a combination of a complete PSA with an incomplete superficial femoral artery (SFA) - this type can be subcategorised into type 2a where the SFA is present but does not communicate with the popliteal artery and type 2b, where the SFA is absent. Type 3 describes a normal femoral artery with an incomplete proximal segment of the PSA and with type 4, there is again a normal femoral artery with an incomplete distal segment of the PSA ${ }^{[5]}$.

A further type was later added: Type 5, where the PSA originates from the median sacral artery and this is subcategorised further into type 5a, where there is a developed SFA and type $5 b$, where there is an underdeveloped SFA ${ }^{[6]}$. Other classification systems have also been proposed (see Figure 4).

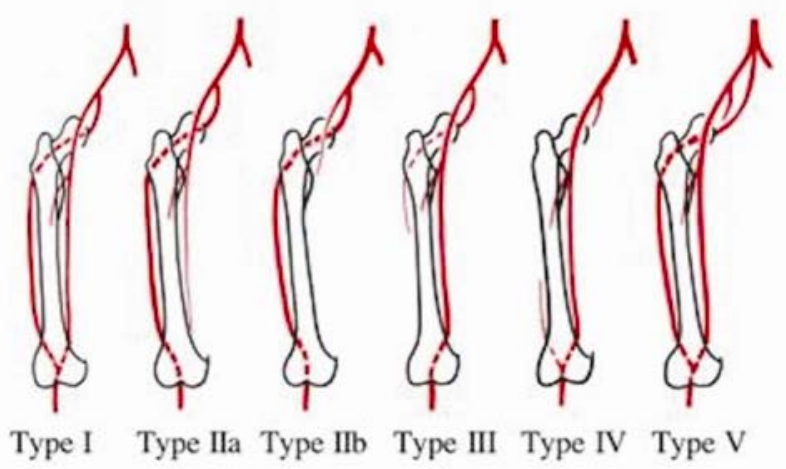

Figure 4. Types of Persistent Sciatic System ${ }^{[3]}$

Approximately $48 \%$ of PSAs are complicated with a PSA aneurysm, suggesting an overall incidence of $0.015 \%-0.03 \%{ }^{[2]}$. Aneurysmal changes are most often hypothesed secondary to trauma as anatomically the position is vulnerable to this. 
Henceforth a detailed trauma history is imperative as well as bearing in mind the other differentials which include femoral artery pathologies, gluteal artery aneurysm and arteriovenous fistulas. The majority of PSA aneurysms is asymptomatic but can present clinically with symptoms of limb ischaemia due to thrombosis or distal embolisation, a pulsatile gluteal mass or lower limb neurology due to compression of the sciatic nerve.

Treatment for a PSA aneurysm can be similar to that of other peripheral arterial aneurysms. This includes endovascular stenting, coil embolization which may also require revascularisation and surgical exclusion bypass grafting ${ }^{[7]}$. An open surgical approach is usually undertaken through a postero/posterolateral approach and adequate exposure is essential due to the risk of iatrogenic injury to the sciatic nerve. Aneurysms can be excluded or excised. In cases of incomplete persistent sciatic artery, exclusion by distal and proximal ligation to the aneurysm with a bypass graft for revascularization is the mainstay of treatment if the patient is fit for surgery. Endovascular treatment offers the advantage to the more co-morbid patients and avoids added revascualrisation procedures, however, suitability for endovascular repair depends on the morphology of the aneurysm and the distal vascular supply. The durability and longevity of such approaches is not clear ${ }^{[8]}$.

\section{References}

[1] Martin KW, Heyde GL, McCready RA, et al. Sciatic artery aneurysms: report of three cases and review of the literature. J Vasc Surg. 1986; 4: 365-371. http://dx.doi.org/10.1016/0741-5214(86)90232-6

[2] Van Hooft IM, Zeebregts CJ, van Sterkenburg SM, et al. The persistent sciatic artery. Eur J Vasc Endovasc Surg. 2009 ; 37: 85-91. PMid:19231248 http://dx.doi.org/10.1016/j.ejvs.2009.01.014

[3] Shutze W P, Garrett W V, Smith B L. Persistent sciatic artery: collective review and management. Annals of Vascular Surgery. 1993; 7(3): 303-310. PMid:8318397 http://dx.doi.org/10.1007/BF02000260

[4] Hassan A. Symptomatic persistent sciatic artery. J Am Coll Surg. 2004; 199: 171-3. PMid:15217650 http://dx.doi.org/10.1016/j.jamcollsurg.2004.02.020

[5] Pillet J, Cronier P, Mercier P, et al. The ischio popliteal arterial trunk: a report of two cases. Anat Clin. 1982 ; 3: 329-31. http://dx.doi.org/10.1007/BF01798943

[6] Gauffre S, Lasjaunias P, Zerah M. Sciatic artery: a case, review of literature and attempt of systematization. Surg Radiol Anat. 1994; 16 (1): 105-9. PMid:8047957 http://dx.doi.org/10.1007/BF01627932

[7] Brantley SK, Rigdon EE, Raju S. Persistent sciatic artery: embryology, pathology, and treatment. J Vasc Surg. 1993; $18: 242-8$. http://dx.doi.org/10.1016/0741-5214(93)90604-K

[8] Brasileiro JL, Chen J, Santos MA. Persistent sciatic artery aneurysm: case report. J. vasc. bras. 2008 Mar; 7(1). 